COMMENT. Histochemical staining of cytochrome $c$ oxidase may be used for the diagnosis of mitochondrial myopathy when ragged red fibers are lacking. Schon EA et al. and Munnich A et al. review the mitochondrial myopathies and the clinical aspects of mitrochondrial disorders in the current issue of International Pediatrics 1992; 7:23-33. The diagnosis of mitochondrial disorder should be considered with (1) an unexplained association of symptoms; (2) an early onset and a rapidly progressive course; and (3) involvement of unrelated organs which share no common embryologic origin and no common biological functions. Determination of lactate/pyruvate and ketone body molar ratios in plasma may help to select patients at risk for further investigation. In a family with infantile mitrochondrial myopathy and cardiomyopathy reported from Rome, Italy, no correlation was found in the muscle between biochemistry and severity of the clinical intrafamilial phenotype (Bertini E et al. Neurology April 1992; 42(Suppl 3):267).

\title{
DYSTROPHIN IN FETAL MUSCLE
}

Dystrophin, the product of the Duchenne muscular dystrophy gene, was studied in human fetal skeletal muscle from 9 to 26 weeks of gestation at the Jerry Lewis Muscle Research Centre, Hammersmith Hospital, London. Dystrophin was localized to the sarcolemma of myotubes in fetal muscle from at least 9 weeks of gestation. Dystrophin immunostaining increased and became more uniform with age and sarcolemmal staining of myotubes was intense by 26 weeks of gestation. Western blot analysis showed a lower relative molecular mass protein in fetal tissue compared to adult tissue. This persisted until 26 weeks of gestation but switched to the adult form by 9 months of age. The data demonstrated several specific isoforms of dystrophin present in developing skeletal muscle (Clerk A et al. Characterisation of dystrophin during development of human skeletal muscle. Development Feb $1992 ; \underline{114}: 395-402)$.

COMMENT. Tome FMS et al. in Paris, France and Boston, MA report on the distribution and localization of dystrophin and dystrophinrelated protein during skeletal muscle development and changes in Duchenne muscular dystrophy fetuses. Dystrophin of the same size as in adults was present in normal fetuses as soon as 8 weeks gestation in this study. It was absent in Duchenne muscular dystrophy fetuses. The dystrophin-related protein was quantitatively and qualitatively comparable in normal and Duchenne fetuses. The authors concluded that the prenatal diagnosis of Duchenne muscular dystrophy can be confirmed by immunocytochemical or biochemical analysis of muscular dystrophin in aborted fetuses. Dystrophin-related protein has a particular role in synaptic function which is not disturbed in Duchenne muscular dystrophy (Neurology April 1992; 42(Suppl 3):227-228). 